\title{
Molecular Frame Reconstruction Using Time-Domain Photoionization Interferometry
}

\author{
Claude Marceau, ${ }^{1}$ Varun Makhija, ${ }^{2}$ Dominique Platzer, ${ }^{1}$ A. Yu. Naumov,${ }^{1}$ P. B. Corkum, ${ }^{1}$ \\ Albert Stolow, ${ }^{2,3,4}$ D. M. Villeneuve, ${ }^{1}$ and Paul Hockett ${ }^{4, *}$ \\ ${ }^{1}$ Joint Attosecond Science Laboratory, National Research Council of Canada and University of Ottawa, \\ 100 Sussex Drive, Ottawa, Ontario K1A OR6, Canada \\ ${ }^{2}$ Department of Physics, University of Ottawa, 150 Louis Pasteur, Ottawa, Ontario K1N 6N5, Canada \\ ${ }^{3}$ Department of Chemistry, University of Ottawa, 10 Marie Curies, Ottawa, Ontario K1N 6N6, Canada \\ ${ }^{4}$ National Research Council of Canada, 100 Sussex Drive, Ottawa, Ontario K1A OR6, Canada
}

(Received 30 January 2017; published 22 August 2017)

\begin{abstract}
Photoionization of molecular species is, essentially, a multipath interferometer with both experimentally controllable and intrinsic molecular characteristics. In this work, XUV photoionization of impulsively aligned molecular targets $\left(\mathrm{N}_{2}\right)$ is used to provide a time-domain route to "complete" photoionization experiments, in which the rotational wave packet controls the geometric part of the photoionization interferometer. The data obtained is sufficient to determine the magnitudes and phases of the ionization matrix elements for all observed channels, and to reconstruct molecular frame interferograms from lab frame measurements. In principle, this methodology provides a time-domain route to complete photoionization experiments and the molecular frame, which is generally applicable to any molecule (no prerequisites), for all energies and ionization channels.
\end{abstract}

DOI: 10.1103/PhysRevLett.119.083401

Photoionization is an interferometric process in which the final observable results from a coherent sum over multiple quantum paths to a set of final continuum photoelectron states $|k, l, m\rangle[1,2]$. Interferences between these components are manifest in the observable energy spectra and photoelectron angular distributions (PADs), the latter of which can be considered as a particularly high information-content observable, extremely sensitive to the phases of the partial waves $|l, m\rangle[2-5]$; consequently, PADs have been investigated in a large range of control and metrology scenarios $[6,7]$. In the context of phase-sensitive metrology, the goal is to obtain the full set of complex photoionization matrix elements and, hence, characterize the photoelectron wave function, by analysis of sets of PAD measurementsthis is a "complete" photoionization experiment $[8,9]$.

In the molecular case, the number of final $|l, m\rangle$ states is typically large, and obtaining a sufficient data set for a complete experiment remains a challenge. In the energy domain, a number of different schemes have been demonstrated in both the laboratory (LF) and molecular frames (MF) [10-16]. The common theme to all these measurements is some form of control over the experimental contributions to the photoionization interferometer (e.g., rotational state, polarization geometry), to which the intrinsic molecular contributions remain invariant. Limitations of previous attempts have been the ability to obtain a sufficiently large data set and molecular specificity in the methodologies, i.e., prerequisites such as low density of states [14], resonances [15], or dissociative channels [11-13].

In the time domain, rotational wave packets can be utilized to control the geometric part of the interferometer. In this case, a high degree of spatiotemporal control of the axis distribution (alignment) of the ionizing molecular ensemble in the LF can be obtained via preparation of a broad rotational wave packet with IR laser pulses. Hence, at any given time delay of the ionizing laser pulse, a different molecular alignment and set of polarization geometries are coherently probed. Although this idea is conceptually obvious, the theory is complex; it has been elucidated by multiple authors (e.g., Refs. [5,17-21]), but-to date-there have been no experimental demonstrations beyond the limiting case of a narrow wave packet prepared via resonant excitation $[15,19,22]$ (cf. rotational coherence spectroscopy [23,24]) and an exploration in the related case of high-harmonic spectroscopy from an impulsively aligned sample [25]. While experimental methods for preparing rotational wave packets and measuring PADs are relatively well established $[6,19,26,27]$, the analysis of such experiments remains challenging, since both the rotational wave packet and the photoionization dynamics must now be fully characterized. However, the benefits are significant-the time-domain methodology is, in principle, completely general with no molecule-specific prerequisites; additionally the use of high harmonics for ionization provides channel and energy multiplexing in each time-domain measurement, resulting in an extremely high information-content metrology $[5,27,28]$. Furthermore, if the determination of the photoionization matrix elements is of sufficient fidelity, one can reconstruct the MF interferograms without the necessity for direct MF measurements. In general, such measurements are desirable for detailed understanding of molecular processes [29].

Here we present a general time-domain approach to complete photoionization experiments in molecules. Experimentally, a double-pulse impulsive alignment scheme 
is used to create a broad rotational wave packet in $\mathrm{N}_{2}$ [30-32]. High harmonics of a 267-nm driving field are used to ionize the aligned ensemble, and velocity-map imaging (VMI) provides energy-, angle-, and time-resolved photoelectron interferograms. A bootstrapping methodology is employed to analyze the data, in which (i) the prepared rotational wave packet is characterized without knowledge of the intrinsic molecular photoionization dynamics [33] and (ii) these results feed into a protocol for the determination of the channel-specific ionization matrix elements. As a test of the results so obtained, the MF interferograms (as a function of polarization geometry) are reconstructed and compared with $a b$ initio calculations. An outline of the method and the key results are presented herein for the general reader; interested readers can obtain an extended presentation and discussion, including numerical data and analysis codes, in the Supplemental Material [34]. This repository is broadly in line with Open Science Transparency and Openness Promotion guidelines [55], and it is hoped that this provides a foundation for other investigators to explore and build on the methodology presented herein.

Experiment.-Laser pulses were generated by a Ti:sapphire amplifier $(100 \mathrm{~Hz}, 11 \mathrm{~mJ}, 800 \mathrm{~nm}$, and $50 \mathrm{fs}$ ). Approximately $3.5 \mathrm{~mJ}$ was used to generate the third harmonic ( $267 \mathrm{~nm}, \sim 0.05 \mathrm{~mJ}$, and $\sim 100 \mathrm{fs}$ ) via double and sum mixing stages in $\beta$-barium borate. The output was filtered by dichroic mirrors and then sent into a vacuum chamber, where high harmonics were generated from a pulsed gas jet of argon. Harmonics were then sent through a 200-nm-thick aluminum foil to filter out the driving laser light; the final spectrum was dominated by harmonics $5(\mathrm{H} 5$, $h \nu=23.3 \mathrm{eV})$ and $7(\mathrm{H} 7, h \nu=32.6 \mathrm{eV})$. The remainder of the initial $800-\mathrm{nm}$ beam $(7.5 \mathrm{~mJ})$ was sent to a Michelson interferometer; the two replicas of the pulse generated formed alignment pulses. The alignment and filtered harmonic beams were recombined (on a holey mirror) and focused into the interaction region of a VMI spectrometer [26]. Spatial and temporal superposition of the alignment pulses and the harmonic pulses was achieved by maximizing the ac Stark shifts in the photoelectron spectrum of argon. The estimated laser parameters in the interaction region for each alignment pulse are $0.5 \mathrm{~mJ}$ and $100 \mathrm{fs}$ at focus, resulting in a peak intensity $I=20 \mathrm{TW} / \mathrm{cm}^{2}$. All beams were linearly polarized, and the data reported herein was obtained for a parallel polarization geometry [Fig. 1(a)]. The delay between the two pump pulses was experimentally optimized (for maximal alignment) at $3.76 \mathrm{ps}$, near the rising edge of the half revival induced by the first pulse [32].

Figure 1 presents three example photoelectron images from molecular nitrogen (99.999\% purity, 4.8 bar backing pressure, $250 \mu \mathrm{m}$ nozzle) and corresponding photoelectron spectra. The complete data set consists of 150 temporal steps in 67-fs increments from $t=-0.5 \mathrm{ps}$ to $+9.5 \mathrm{ps}$, where $t=0$ is defined as the peak intensity of the second alignment pulse. On average, about 30000 laser shots were
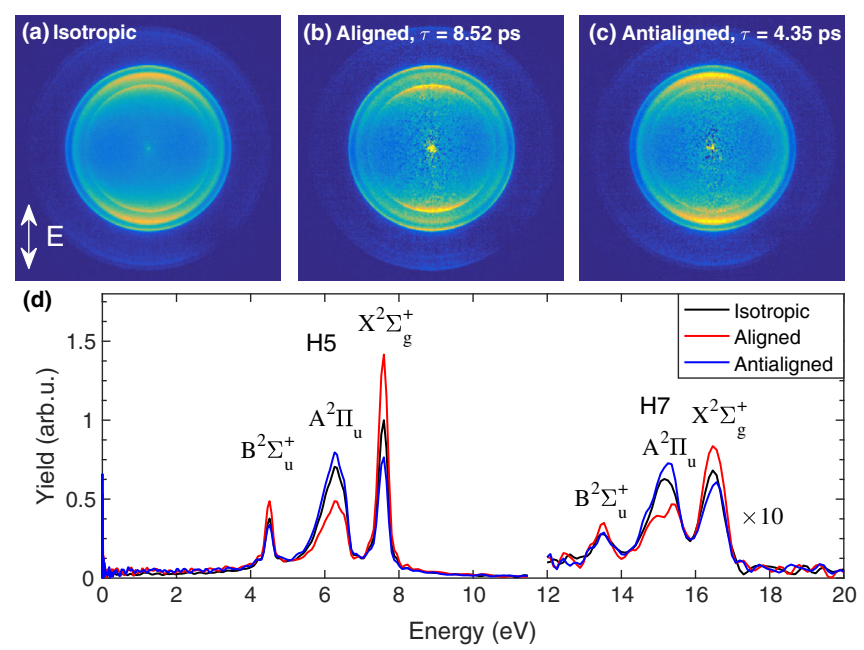

FIG. 1. VMI photoelectron images from (a) isotropic (no alignment pulses), (b) aligned, and (c) antialigned nitrogen molecules. The polarization of both alignment pulses and the XUV pulse are linear as indicated in (a). (d) The electron yields; labels provide cationic state assignments for the observed features.

accumulated at each delay. Additional signals that monitored gas density in the VMI, harmonic source brightness, and background contributions (above threshold ionization from the alignment pulses, scattered light, and residual background gas signals) were taken together with the data, providing calibration and background measurements.

The observed angular distributions are most generally expressed as an expansion in spherical harmonics,

$$
S\left(\theta_{e}, \phi_{e}, t\right)=\sum_{L, M} \beta_{L, M}(t) Y_{L, M}\left(\theta_{e}, \phi_{e}\right),
$$

where $\beta_{L, M}(t)$ are the expansion coefficients, and $\theta_{e}$ and $\phi_{e}$ are polar and azimuthal ejection angles of the electron respectively. For cylindrically symmetric cases, which applies to all LF quantities discussed herein, $M=0$; hence, the angular dependence is reduced to a function of $\theta$ and the angle $\phi$ is redundant. The LF symmetry also restricts terms to even $L$ only. The experimental data analysis consisted of inverting each background-subtracted VMI image using the pBaseX algorithm [56], providing expansion coefficients for each photoelectron image as a function of radius, subsequently averaged over each photoelectron band [Fig. 1(d)] to provide $\beta_{L, M}(t)$ for each ionization channel; this is hereafter denoted as the $X, A$, and $B$ channels, referring to ionization into the ground, first, and second excited states of the ion (see Fig. 1). Selected time-domain results are shown in Figs. 2 and 3 for the H5 $X$ channel (full presentation in the Supplemental Material [34]).

Theory.-The $\beta_{L, M}(t)$ can further be expanded in terms of the contributing physical factors,

$$
\beta_{L, M}(t)=\sum_{K, Q}\left(\sum_{\alpha, \alpha^{\prime}} \gamma_{K, Q}^{\alpha, \alpha^{\prime}} \boldsymbol{D}_{\alpha}^{*} \boldsymbol{D}_{\alpha^{\prime}}\right) A_{K,-Q}(t) .
$$


In this form, all of the angular momentum coupling terms are denoted by $\gamma$ and can be defined analytically. $A_{K,-Q}(t)$ are the time-dependent axis distribution moments (ADMs) arising from the rotational wave packet and define the spatial alignment of the molecular ensemble: the full axis distribution in the LF can be described as $P(\theta, \phi, t)=$ $\sum_{K, Q} A_{K,-Q}(t) Y_{K, Q}(\theta, \phi)$ [cf. Eq. (1)], where $\theta$ and $\phi$ are polar and azimuthal axis-alignment angles respectively. The ADMs couple geometrically to the ionization dynamics - the intricate details of this coupling dictates the response of different partial waves to the ADMs, and different revivals in the rotational wave packet are sensitive to different aspects of the ionization dynamics [21]. The $\boldsymbol{D}_{\alpha}$ are the symmetrized ionization matrix elements (complex) [36] to be determined. All other required quantum numbers are denoted $\alpha$, and the coherent summation is obtained by summing over all possible pairs of each quantum number. The full form of Eq. (2), which explicitly shows all summations (all interfering paths) that contribute to each observable $\beta_{L, M}(t)$, is given in the Supplemental Material [34]. This treatment follows the formalism of Underwood and Reid $[17,59,60]$ and is applicable to single-photon ionization in the dipole limit. In the analysis described herein, it is assumed that the ionization matrix elements are constant over each observed photoelectron band, which is expected to be a reasonable approximation in this case. In cases where this approximation does not hold, the retrieved matrix elements will be averaged over any underlying structure or dynamics (e.g., the range of nuclear geometries probed or continuum resonances) [18].

Evidently, the above equation can be recast as

$$
\beta_{L, M}(t)=\sum_{K, Q} C_{K, Q}^{L, M} A_{K,-Q}(t),
$$

where the coefficients $C_{K, Q}^{L, M}$ contain all the terms in the brackets in Eq. (2). Because this equation is linear in the ADMs, the measured $\beta_{L, M}(t)$ can be used to determine the ionization dynamics in this phenomenological form; such solutions of Eq. (3) constitute the first part of the bootstrapping procedure [33].

Analysis.-The ADMs can be computed by solving the time-dependent Schrödinger equation for a linear rigid rotor in a linearly polarized nonresonant pulse. Although the $C_{K, Q}^{L, M}$ are channel dependent, the axis distribution is universal; hence, fits to different $L$ and/or ionization channels can be performed as a rigorous cross-check on the results. In this case, computation of the ADMs was carried out for rotational temperatures $T_{\text {rot }}=1$ to $30 \mathrm{~K}$ in $1 \mathrm{~K}$ steps and laser intensities $I=10$ to $30 \mathrm{TW} / \mathrm{cm}^{2}$ in $2 \mathrm{TW} / \mathrm{cm}^{2}$ steps for each pulse. The delay between pulses and their durations were kept fixed to the experimentally determined values. The calculated ADMs were stored and a linear regression to solve Eq. (3) was carried out for each parameter set on the measured $\beta_{0,0}(t), \beta_{2,0}(t)$, and $\beta_{4,0}(t)$ parameters for the $X$ channel. Each regression was performed independently, and $I=20 \mathrm{TW} / \mathrm{cm}^{2}$ for each pulse and $T_{\text {rot }}=15 \mathrm{~K}$ provided the best fit in all three cases, in good agreement with the experimentally estimated values. The fits are shown in Fig. 2, along with a comparison with $\beta_{6,0}(t)$ obtained using the same parameter set ( $I$ and $T_{\text {rot }}$ ). Given the fidelity of the fits, the ADMs $\left(K_{\max }=6\right)$ obtained for these parameters are assumed to accurately describe the experimentally prepared axis distributions, and the resultant axis distribution $P(\theta, t)$ is shown in Fig. 2 .

Although the retrieved coefficients $C_{K, Q}^{L, M}$ contain information on the ionization dynamics [33], it is preferable to use the resulting ADMs within the full ionization framework of Eq. (2) to directly retrieve the intrinsic molecular ionization dynamics defined by $\boldsymbol{D}_{\alpha}$. This constitutes the second stage of the bootstrapping procedure. The multiple interfering pathways set the requirement for a high-information-content data set, and the equations are highly nonlinear multivariate quadratic equations. Careful sampling of the solution hyperspace is required in order to ensure unique results: this step was therefore broken down into substeps to allow (a) statistical sampling and (b) further bootstrapping. In (a) repeated coarse fits with minimal information content (selected experimental measurements with distinct ADMs) and randomized seed parameters were employed [61], while (b) took the best-fit result(s) as seeds for fits with higher information content and/or more stringent convergence criteria, based on computational time and desired precision.

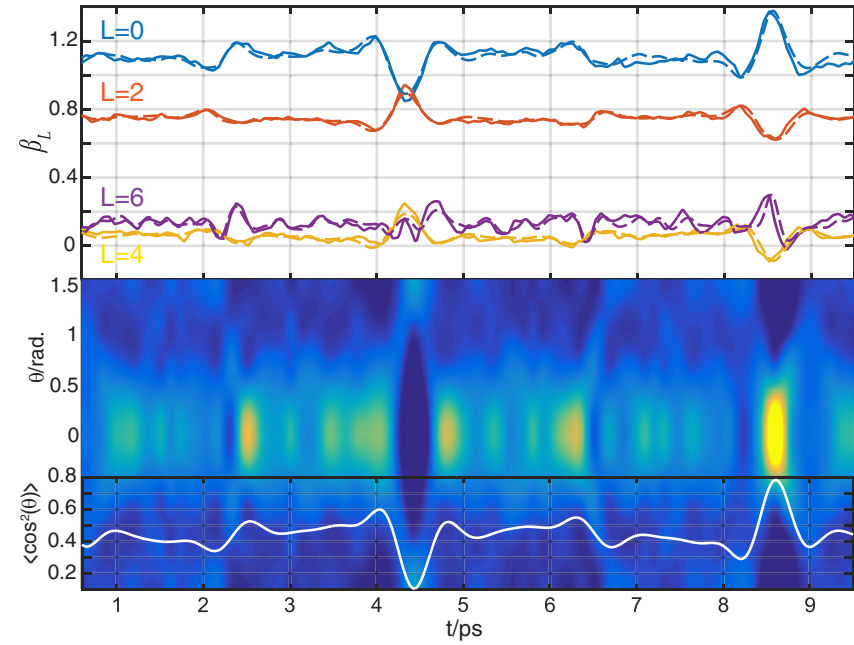

FIG. 2. Molecular alignment determination. (Top) Experiment (solid lines) and fit results (dashed) to Eq. (3), for $L=0,2,4,6$ (Legendre polynomial expansion). (Bottom) Calculated axis distribution $P(\theta, t)$ determined from the fit $\left(I=20 \mathrm{TW} / \mathrm{cm}^{2}\right.$, $T_{\text {rot }}=15 \mathrm{~K}$ ); the inset shows $\left\langle\cos ^{2}(\theta, t)\right\rangle$ for this distribution (a commonly used indicator of the degree of alignment achieved $[21,57,58])$. The temporal axis is defined by the second pulse $(t=0)$, and shows the $1 / 2$ and full revivals at the expected delays of 4.2 and 8.4 ps, respectively, as well as numerous higher-order structures indicating the excitation of a broad angular momentum wave packet. 


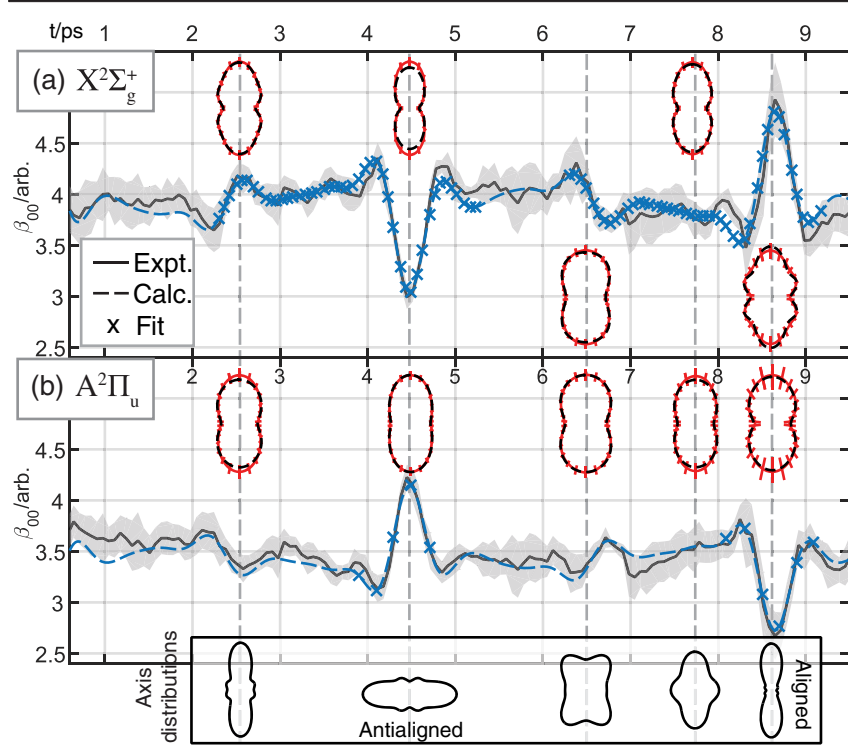

FIG. 3. Fitting of the time-series data. Main panels show the calculation results from Eq. (2) for (a) the $X$ - and (b) $A$-channel yields, $\beta_{00}(t)$, where $\mathrm{x}$ marks points included in the fit; full $\beta_{L, M}(t)$ plots are presented in the Supplemental Material [34]. Examples of the full angle-resolved interferograms $S(\theta, t)$, experimental results (including error bars) and calculated, are also shown, and corresponding axis distributions $P(\theta, t)$ (bottom).

Figure 3 illustrates this method and example results. The $X$ channel is particularly sensitive to the axis distribution, with significant changes in both the yields and angular distributions. In this case, two unique fit results (sets of matrix elements $\boldsymbol{D}_{\alpha}$ ) were obtained via statistical sampling at a coarse level (11 temporal points over the revival features), and fine-tuning via the bootstrapping methodology outlined above (finally incorporating 89 temporal points), led to a single best-fit solution [six complex-valued matrix elements, results as shown in Fig. 3(a)]. For the $A$ and $B$ channels, the data becomes increasingly noisy as the yields decrease, and also indicates much less dramatic dependence on the axis distribution. In these cases, adequate fits were obtained at the coarse level [as indicated in Fig. 3(b)], but further bootstrapping was not explored in either case. For the $A$ channel, three best-fit parameter sets were obtained (seven matrix elements), differing only in the perpendicular continuum waves. For the $B$ channel data, a single best-fit parameter set was obtained (five matrix elements), although four additional parameter sets were within $1 \%$ of the best fit (defined by the minimal residual, $\chi^{2}$ [62]), and differed in the parallel continuum. In these cases, additional data and/or crosschecks on the determined matrix elements are therefore desirable to confirm their validity. The full set of fit results and matrix elements determined (including the associated uncertainties) are given in the Supplemental Material [34].

Finally, from the matrix elements obtained, the MF photoelectron interferograms may be calculated and compared with $a b$ initio results. In this case, the interferograms are no longer restricted by the LF symmetry; hence, this provides a sensitive test of the retrieved matrix elements. Figure 4 shows these results and comparison with ab initio results obtained using ePolyScat $[63,64]$. Results are shown for three different polarization geometries in the MF. The parallel and perpendicular cases cleanly separate sets of matrix elements $\boldsymbol{D}_{\alpha}$ by symmetry, while the diagonal result mixes these components and provides a particularly stringent test of the results [13]. Compared to the LF, the MF results show a wealth of structure. The experimentally reconstructed and computed results for the $X$ channel are in good agreement, while for the $A$ channel the agreement is variable, consistent with larger uncertainties in the retrieved matrix elements (see Ref. [34] for full numerical results). Photoionization of $\mathrm{N}_{2}$ has attracted much interest due to the $\sigma$ continuum shape resonance [65]. Significantly, the MF results (Fig. 4) for the $X$ and $B \sigma$ continua are similar to direct MF measurements in the same photoelectron energy region presented in Refs. [11,66] for 2 and $9 \mathrm{eV}$ photoelectrons; while those studies investigated dissociative core ionization, the scattering and shape-resonance behavior of the $\sigma$ continuum accessed is analogous.

From the $a b$ initio perspective, photoionization calculations present a formidable challenge, and there is a dearth of MF measurements for comparison and validation due to the experimental difficulty of such measurements; thus, the MF results herein can alternatively be viewed as an excellent test for theory $[13,65,67-69]$. From this perspective, consistency between the $a b$ initio and experimental reconstructions can be taken as a good indicator that both methodologies are robust.

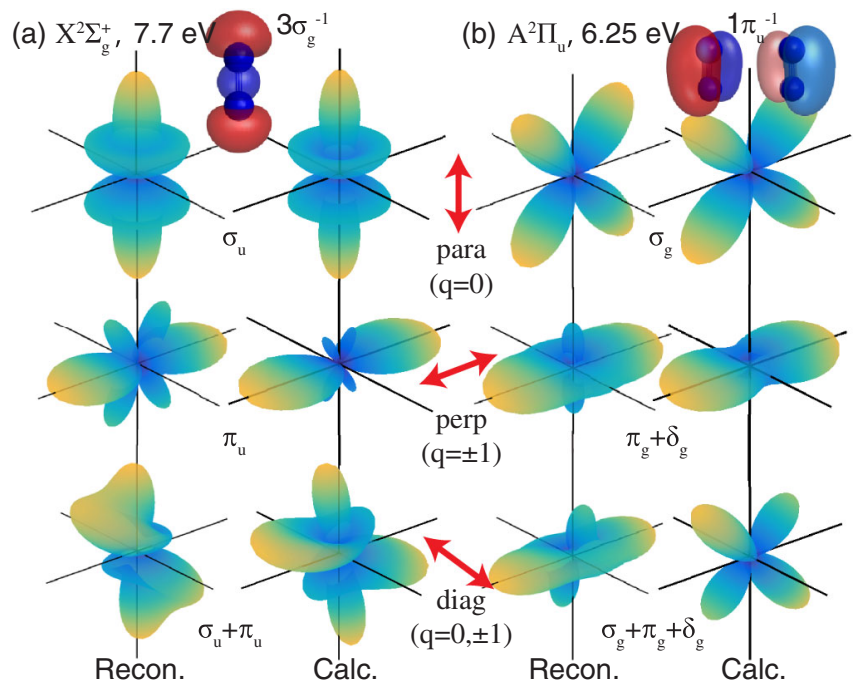

FIG. 4. Molecular frame reconstruction as a function of ionizing orbital $(a, b)$ and polarization geometry (rows). "Recon." indicates MF PADs reconstructed from the experimental analysis; "Calc."represents those determined from ab initio calculations. Each panel also shows the ionizing orbital, and labels indicate the MF polarization geometry (photon projection $q$ ) and corresponding symmetries for the ionization continua accessed. 
In this Letter, time-domain measurements, utilizing molecular alignment techniques, have been demonstrated as a means to "complete" photoionization studies. The timedomain data provided sufficiently high information content to reliably extract the axis distributions and matrix elements for three different ionization channels. A bootstrapping fitting methodology provided a flexible and robust analysis route, and MF reconstructions provided an additional stringent test of the physical parameters so determined, as well as an illustration of their predictive power. The main advantage of this methodology is that it is completely general in principle (although it may be restricted by symmetry in certain cases, and may require noncylindrically symmetric polarization geometries [34]), and is applicable to any molecular ionization problem, provided that the preparation and propagation of the rotational wave packet remains decoupled from other molecular dynamics. All relative magnitudes and phases of the ionization matrix elements can be determined and MF interferograms can be obtained without experimentally challenging MF measurements, which require molecular orientation or fragmentation. The disadvantage is that this fitting methodology is not a black-box procedure, and requires detailed analysis (see Supplemental Material [34]). While this methodology has been demonstrated herein for the simplest case of a homonuclear linear molecule, the theory and analysis protocol allows for arbitrary asymmetric molecules. Equation (3) can provide a sense of the limitations of this. The degree of rotational excitation determines the number of $C_{K, Q}^{L, M}$ that can be extracted from the data. These then provide a set of multivariate quadratic equations in the dipole matrix elements. The nature and solubility of this equation set for asymmetric top molecules will be the subject of a future publication [70]. Some improvements may be possible here, for example, the use of other fitting algorithms (genetic algorithms and homotopy methods [71]), which may allow for a more robust approach less sensitive to local minima in the solution hyperspace, and the use of graphics processing units (GPUs) to massively parallelize the computations.

Experimentally, the use of an extended harmonic spectrum would allow for the observation and analysis of many more photoelectron bands [27], thus providing a route to obtaining even higher degrees of multiplexing in the measurements and MF reconstruction for a range of channels and energies from a single time-domain experimental data set. In this light, the generality of the method suggests such measurements, combined with high-repetition rate laser sources, provide a route to highly multiplexed "complete" experiments and MF reconstruction for a range of molecules, and a tractable method for high-resolution dynamical MF imaging [72], which is otherwise typically restricted by experimental prerequisites and data-acquisition time scales [73].
We thank the U.S. Army Research Office for funding under Contract No. W911NF-14-1-0383. A. S. thanks the NSERC Discovery program for financial support.

paul.hockett@nrc.ca

[1] H. D. Cohen and U. Fano, Phys. Rev. 150, 30 (1966).

[2] D. Dill, J. Chem. Phys. 65, 1130 (1976).

[3] J. Cooper and R. N. Zare, in Lectures in Theoretical Physics: Atomic Collision Processes, edited by S. Geltman, K. T. Mahanthappa, and W. E. Brittin (Gordon and Breach, New York, 1969), Vol. XI-C, pp. 317-337.

[4] K. L. Reid, Annu. Rev. Phys. Chem. 54, 397 (2003).

[5] S. Ramakrishna and T. Seideman, J. Phys. B 45, 194012 (2012).

[6] K. L. Reid, Mol. Phys. 110, 131 (2012).

[7] P. Hockett, C. Lux, M. Wollenhaupt, and T. Baumert, Phys. Rev. A 92, 013412 (2015).

[8] H. Klar and H. Kleinpoppen, J. Phys. B 15, 933 (1982).

[9] U. Becker, J. Electron Spectrosc. Relat. Phenom. 96, 105 (1998).

[10] K. L. Reid, D. J. Leahy, and R. N. Zare, Phys. Rev. Lett. 68, 3527 (1992).

[11] E. Shigemasa, J. Adachi, M. Oura, and A. Yagishita, Phys. Rev. Lett. 74, 359 (1995).

[12] O. Geßner, Y. Hikosaka, B. Zimmermann, A. Hempelmann, R. R. Lucchese, J.H. D. Eland, P.-M. Guyon, and U. Becker, Phys. Rev. Lett. 88, 193002 (2002).

[13] R. R. Lucchese, A. Lafosse, J. C. Brenot, P. M. Guyon, J. C. Houver, M. Lebech, G. Raseev, and D. Dowek, Phys. Rev. A 65, 020702 (2002).

[14] P. Hockett, M. Staniforth, K. L. Reid, and D. Townsend, Phys. Rev. Lett. 102, 253002 (2009).

[15] Y. Tang, Y.-I. Suzuki, T. Horio, and T. Suzuki, Phys. Rev. Lett. 104, 073002 (2010).

[16] P. Hockett, M. Wollenhaupt, C. Lux, and T. Baumert, Phys. Rev. Lett. 112, 223001 (2014).

[17] J. G. Underwood and K. L. Reid, J. Chem. Phys. 113, 1067 (2000).

[18] T. Seideman, Annu. Rev. Phys. Chem. 53, 41 (2002).

[19] T. Suzuki, Annu. Rev. Phys. Chem. 57, 555 (2006).

[20] Y.-I. Suzuki and T. Suzuki, Mol. Phys. 105, 1675 (2007).

[21] P. Hockett, New J. Phys. 17, 023069 (2015).

[22] Y.-I. Suzuki, Y. Tang, and T. Suzuki, Phys. Chem. Chem. Phys. 14, 7309 (2012).

[23] P. M. Felker and A. H. Zewail, J. Chem. Phys. 86, 2460 (1987).

[24] P. M. Felker, J. Phys. Chem. 96, 7844 (1992).

[25] R. M. Lock, S. Ramakrishna, X. Zhou, H. C. Kapteyn, M. M. Murnane, and T. Seideman, Phys. Rev. Lett. 108, 133901 (2012).

[26] A. T. J. B. Eppink and D. H. Parker, Rev. Sci. Instrum. 68, 3477 (1997).

[27] A. Rouzée, F. Kelkensberg, W. K. Siu, G. Gademann, R. R. Lucchese, and M. J. J. Vrakking, J. Phys. B 45, 074016 (2012).

[28] Y.-I. Suzuki and T. Seideman, J. Chem. Phys. 122, 234302 (2005).

[29] R. R. Lucchese and A. Stolow, J. Phys. B 45, 190201 (2012).

[30] C. Z. Bisgaard, M. D. Poulsen, E. Péronne, S. S. Viftrup, and H. Stapelfeldt, Phys. Rev. Lett. 92, 173004 (2004). 
[31] J. P. Cryan, P. H. Bucksbaum, and R. N. Coffee, Phys. Rev. A 80, 063412 (2009).

[32] X. Ren, V. Makhija, A.-T. Le, J. Troß, S. Mondal, C. Jin, V. Kumarappan, and C. Trallero-Herrero, Phys. Rev. A 88, 043421 (2013).

[33] V. Makhija, X. Ren, D. Gockel, A.-T. Le, and V. Kumarappan, arXiv:1611.06476.

[34] See Supplemental Material at http://link.aps.org/ supplemental/10.1103/PhysRevLett.119.083401 for an extended presentation and discussion, including the complete formalism applied, a full list of retrieved matrix elements, numerical data and analysis codes, which includes Refs. [35-54].

[35] A. Messiah, Quantum Mechanics (North-Holland Publishing Company, Amsterdam, 1970), Vol. I.

[36] N. Chandra, J. Phys. B 20, 3405 (1987).

[37] K. L. Reid, D. J. Leahy, and R. N. Zare, J. Chem. Phys. 95, 1746 (1991).

[38] H. Park and R. N. Zare, J. Chem. Phys. 104, 4554 (1996).

[39] K. L. Reid and I. Powis, J. Chem. Phys. 100, 1066 (1994).

[40] R. R. Lucchese, G. Raseev, and V. McKoy, Phys. Rev. A 25, 2572 (1982).

[41] Y. Arasaki, K. Takatsuka, K. Wang, and V. McKoy, Chem. Phys. Lett. 302, 363 (1999).

[42] D. J. Leahy, K. L. Reid, and R. N. Zare, J. Chem. Phys. 95, 1757 (1991).

[43] Y.-Y. Yin, C. Chen, D. S. Elliott, and A. V. Smith, Phys. Rev. Lett. 69, 2353 (1992).

[44] Y.-Y. Yin, D. S. Elliott, R. Shehadeh, and E. R. Grant, Chem. Phys. Lett. 241, 591 (1995).

[45] S. Ramakrishna and T. Seideman, Phys. Rev. A 87, 023411 (2013).

[46] T. Seideman and S. C. Althorpe, J. Electron Spectrosc. Relat. Phenom. 108, 99 (2000).

[47] T. Seideman, Phys. Rev. A 64, 042504 (2001).

[48] J. López-Domínguez, D. Hardy, A. Das, E. Poliakoff, A. Aguilar, and R. R. Lucchese, J. Electron Spectrosc. Relat. Phenom. 185, 211 (2012).

[49] A. Osterwalder, M. J. Nee, J. Zhou, and D. M. Neumark, J. Chem. Phys. 121, 6317 (2004).

[50] P. Hockett, Ph.D. thesis, University of Nottingham, 2009, http://eprints.nottingham.ac.uk/10857/.
[51] P. Hockett, C. Z. Bisgaard, O. J. Clarkin, and A. Stolow, Nat. Phys. 7, 612 (2011).

[52] P. Hockett, DOI: 10.6084/m9.figshare.3545639.v1 (2016).

[53] P. Hockett, arXiv:1611.04043.

[54] N. Barnes, Nature (London) 467, 753 (2010).

[55] B. A. Nosek et al., Science 348, 1422 (2015).

[56] G. A. Garcia, L. Nahon, and I. Powis, Rev. Sci. Instrum. 75, 4989 (2004).

[57] M. Artamonov and T. Seideman, J. Chem. Phys. 128, 154313 (2008).

[58] V. Makhija, X. Ren, and V. Kumarappan, Phys. Rev. A 85, 033425 (2012).

[59] K. L. Reid and J. G. Underwood, J. Chem. Phys. 112, 3643 (2000).

[60] A. Stolow and J. G. Underwood, in Advances in Chemical Physics (Wiley-Blackwell, New York, 2008), pp. 497-584.

[61] P. Hockett, M. Wollenhaupt, C. Lux, and T. Baumert, Phys. Rev. A 92, 013411 (2015).

[62] P. R. Bevington and D. K. Robinson, Data Reduction and Error Analysis for the Physical Sciences, 2nd ed. (McGrawHill, New York, 1992).

[63] F. A. Gianturco, R. R. Lucchese, and N. Sanna, J. Chem. Phys. 100, 6464 (1994).

[64] A. P. P. Natalense and R. R. Lucchese, J. Chem. Phys. 111, 5344 (1999).

[65] R. R. Lucchese, K. Takatsuka, and V. McKoy, Phys. Rep. 131, 147 (1986).

[66] A. Yagishita, K. Hosaka, and J.-I. Adachi, J. Electron Spectrosc. Relat. Phenom. 142, 295 (2005).

[67] S. K. Semenov, N. A. Cherepkov, G. H. Fecher, and G. Schönhense, Phys. Rev. A 61, 032704 (2000).

[68] P. Lin and R. R. Lucchese, J. Chem. Phys. 117, 4348 (2002).

[69] C. Jin, A.-T. Le, S.-F. Zhao, R. R. Lucchese, and C. D. Lin, Phys. Rev. A 81, 033421 (2010).

[70] V. Makhija and P. Hockett (to be published).

[71] A. J. Sommese and C. W. Wampler II, The Numerical Solution of Systems of Polynomials Arising in Engineering and Science (World Scientific Publishing Company, Singapore, 2005).

[72] P. Hockett, DOI: 10.6084/m9.figshare.3580734 (2014).

[73] P. Hockett, E. Ripani, A. Rytwinski, and A. Stolow, J. Mod. Opt. 60, 1409 (2013). 